\title{
Commentary Brachial artery flow velocity variation: another victory for hand-carried ultrasound?
}

\author{
Gregory A Schmidt
}

Department of Internal Medicine, University of lowa, 200 Hawkins Drive, C33-GH, lowa City, IA 52246, USA

Corresponding author: Gregory A Schmidt, gregory-a-schmidt@uiowa.edu

Published: 16 October 2009

Critical Care 2009, 13:195 (doi:10.1186/cc8120)

This article is online at http://ccforum.com/content/13/5/195

(c) 2009 BioMed Central Ltd

See related research by Monge García et al., http://ccforum.com/content/13/5/R142

\begin{abstract}
Dynamic predictors are clearly superior to static pressures in predicting whether a patient will respond to a fluid bolus. Handcarried ultrasound (HCUS) can measure changes in blood flow velocity in the brachial artery that parallel arterial pulse pressure variation. The potential for HCUS to guide fluid therapy noninvasively must overcome problems of sensitivity and applicability.
\end{abstract}

In the past 5 years, hand-carried ultrasound (HCUS) devices have joined the intensivist's toolbox for guiding invasive procedures, for analyzing the pathophysiological basis for circulatory shock, and for predicting fluid responsiveness by revealing inferior vena caval collapsibility. In a recent paper, Monge Garcia and colleagues demonstrated that respiratoryinduced variation in brachial artery peak flow velocity $\left(\Delta\right.$ Vpeak $\left._{\mathrm{BA}}\right)$ could be measured with HCUS and the result predicted the cardiac output response to a fluid challenge [1].

Fluid therapy for critically ill, hemodynamically unstable patients presents clinicians with a dilemma. On the one hand, a fluid bolus may augment cardiac output, improve critical organ perfusion, and even save the patient's life. On the other hand, fluid may confer no hemodynamic benefit, while adding to pulmonary edema, amongst other ills. How often is a fluid bolus harmful, as opposed to helpful? When an intensivist judges that a fluid bolus is necessary, only one-half of patients respond with a meaningful boost in cardiac output [2]. Especially for patients most likely to be harmed (for example, those with concomitant acute lung and kidney injury), knowing whether fluids will enhance perfusion should be clinically valuable.

Historically, clinicians have relied on static hemodynamic parameters, such as the central venous pressure, to judge whether fluids are likely to aid the circulation. A multitude of studies, accumulating for more than two decades, show that the central venous pressure and its more invasive cousin, the pulmonary artery occlusion (or wedge) pressure, are no more reliable than a coin toss in forecasting whether an individual subject will respond positively to a fluid bolus. When seen in subjects with sepsis [3], with acute respiratory failure [4], or following cardiac surgery [5], this lack of predictive accuracy was attributed to effects of surgery or positive end-expiratory pressure on, for example, ventricular compliance. How disturbing, then, to find that the central venous pressure and pulmonary artery occlusion pressure fail to correlate with ventricular volumes or fluid responsiveness even in healthy normal individuals [6]!

In contrast to the failure of static measures, a novel set of predictors that rely on perturbing the circulation accurately foretell whether fluids will augment cardiac output. These dynamic measures generally employ controlled mechanical ventilation to raise the pleural pressure (some alternatively depend on raising the legs, measuring the effect of spontaneous breathing, or altering the positive end-expiratory pressure) and quite accurately predict fluid responsiveness. In the passive patient, the stroke volume varies with ventilation to a degree that reflects whether the ventricles are operating on the rising or flat portion of the Starling function curve. Patients whose circulations can respond to fluids will therefore demonstrate substantially greater cyclical variability in stroke volume. As the stroke volume changes, so vary the systolic pressure [7], the pulse pressure [7], and the aortic blood flow velocity [8]. Similar cardiopulmonary interactions explain that changes in the diameter of the inferior vena cava also predict fluid responsiveness $[9,10]$. At the same time, dynamic predictors have limitations: a regular cardiac rhythm is required; most predictors rely on a fully passive patient ventilated with a tidal volume higher than that usually

$\Delta \mathrm{Vpeak}_{\mathrm{BA}}=$ variation in brachial artery peak flow velocity; $\mathrm{HCUS}=$ hand-carried ultrasound. 
accepted ( 8 to $10 \mathrm{~cm}^{3} / \mathrm{kg}$ ); and many of the measures are invasive, requiring, for example, an arterial catheter.

We have previously shown that $\Delta \mathrm{Vpeak}_{\mathrm{BA}}$ measured by HCUS mirrors the respiratory changes in arterial blood pressure transduced through a radial artery catheter [11], and suggested that this might serve as a non-invasive parameter for gauging fluid responsiveness. Monge García and colleagues have confirmed and extended this work by directly correlating $\Delta \mathrm{Vpeak}_{\mathrm{BA}}$ to the impact of a fluid challenge [1]. Subjects had a regular cardiac rhythm, lacked respiratory efforts (assured by examination of ventilator waveforms and, if signs of effort were seen, by neuromuscular blockade), were ventilated with tidal volumes of 8 to $10 \mathrm{~cm}^{3} / \mathrm{kg}$ ideal body weight, and were judged fluid responsive if the stroke volume index increased by at least $15 \%$ after $500 \mathrm{~cm}^{3}$ colloid. The primary finding was that $\Delta$ Vpeak $_{\mathrm{BA}}>10 \%$ predicted fluid responsiveness with a sensitivity of $74 \%$ and a specificity of $95 \%$.

Three additional findings deserve comment. First, as others have shown, radial artery pulse pressure variation quite accurately predicted the response to fluid, with a value $>10 \%$ being both sensitive and specific (95\% and 95\%). Second, the mean arterial blood pressure increased $13 \mathrm{mmHg}$ in the nonresponders, confirming that this simple vital sign cannot serve as a surrogate for changes in perfusion. Finally, the central venous pressure performed poorly (area under the receiver operator characteristic curve only 0.64).

Dynamic predictors (especially pulse pressure variation) are clearly superior to static pressures, but the role of $\Delta \mathrm{Vpeak}_{\mathrm{BA}}$ is less certain. First, a screening test demands high sensitivity (not specificity). For the clinician to withhold a fluid challenge, the predictor must identify nearly all patients capable of responding, otherwise too many patients will be denied a potentially life-saving therapy. A sensitivity of $74 \%$ does not meet this test. Second, a rapid, non-invasive monitor such as HCUS might have greatest application in the field or very early in resuscitation, before invasive lines are placed. Yet the risk-benefit dilemma posed by fluid bolus is rare in the field renal failure is not established and the likelihood of responding to fluid is surely much higher than the $50 \%$ range typical of intensive care unit patients.

While the study of Monge García and colleagues corroborates the view that fluid responsiveness is best predicted dynamically, further work is needed before $\Delta V_{p e a k_{B A}}$ finds a role in clinical practice.

\section{Competing interests}

The author declares that they have no competing interests.

\section{References}

1. Monge García MI, Gil Cano A, Díaz Monrové JC: Brachial artery peak flow velocity variation to predict fluid responsiveness in mechanically ventilated patients. Crit Care 2009, 13:R142.
2. Durairaj L, Schmidt GA: Fluid therapy in resuscitated sepsis: less is more. Chest 2008, 133:252-263.

3. Calvin JE, Driedger AA, Sibbald WJ: Does the pulmonary capillary wedge pressure predict left ventricular preload in critically ill patients? Crit Care Med 1981, 9:437-443.

4. Lichtwarck-Aschoff M, Zeravik J, Pfeiffer UJ: Intrathoracic blood volume accurately reflects circulatory volume status in critically ill patients with mechanical ventilation. Intensive Care Med 1992, 18:142-147.

5. Hansen RM, Viquerat CE, Matthay MA, Wiener-Kronish JP, DeMarco T, Bahtia S, Marks JD, Botvinick EH, Chatterjee K: Poor correlation between pulmonary arterial wedge pressure and left ventricular end-diastolic volume after coronary artery bypass graft surgery. Anesthesiology 1986, 64:764-770.

6. Kumar A, Anel R, Bunnell E, Habet K, Zanotti S, Marshall S, Neumann A, Ali A, Cheang M, Kavinsky C, Parrillo JE: Pulmonary artery occlusion pressure and central venous pressure fail to predict ventricular filling volume, cardiac performance, or the response to volume infusion in normal subjects. Crit Care Med 2004, 32:691-699.

7. Michard F, Boussat S, Chemla D, Anguel N, Mercat A, Lecarpentier Y, Richard C, Pinsky MR, Teboul JL: Relation between respiratory changes in arterial pulse pressure and fluid responsiveness in septic patients with acute circulatory failure. Am J Respir Crit Care Med 2000, 162:134-138.

8. Feissel M, Michard F, Mangin I, Ruyer O, Faller JP, Teboul JL: Respiratory changes in aortic blood velocity as an indicator of fluid responsiveness in ventilated patients with septic shock. Chest 2001, 119:867-873.

9. Barbier C, Loubières $Y$, Schmit C, Hayon J, Ricôme JL, Jardin F, Vieillard-Baron A: Respiratory changes in inferior vena cava diameter are helpful in predicting fluid responsiveness in ventilated septic patients. Intensive Care Med 2004, 30:17401746.

10. Feissel M, Michard F, Faller JP, Teboul JL: The respiratory variation in inferior vena cava diameter as a guide to fluid therapy. Intensive Care Med 2004, 30:1834-1837.

11. Brennan JM, Blair JE, Hampole C, Goonewardena S, Vasaiwala S, Shah D, Spencer KT, Schmidt GA: Radial artery pulse pressure variation correlates with brachial artery peak velocity variation in ventilated subjects when measured by internal medicine residents using hand-carried ultrasound devices. Chest 2007, 131:1301-1307. 